\title{
A Novel Time-Based Readout Scheme for a Combined PET-CT Detector Using APDs
}

\author{
F. Powolny, E. Auffray, H. Hillemanns, P. Jarron, P. Lecoq, T. C. Meyer, and D. Moraes
}

\begin{abstract}
This paper summarizes CERN R\&D work done in the framework of the European Commission's FP6 BioCare Project. The objective was to develop a novel "time-based" signal processing technique to read out LSO-APD photodetectors for medical imaging. An important aspect was to employ the technique in a combined scenario for both Computer Tomography (CT) and Positron Emission Tomography (PET) with effectively no tradeoffs in efficiency and resolution compared to traditional single mode machines. This made the use of low noise and yet very high-speed monolithic front-end electronics essential so as to assure the required timing characteristics together with a high signal-to-noise ratio. Using APDs for photon detection, two chips, traditionally employed for particle physics, could be identified to meet the above criteria. Although both were not optimized for their intended new medical application, excellent performance in conjunction with LSO-APD sensors could be derived. Whereas a measured energy resolution of $16 \%$ (FWHM) at the $511 \mathrm{keV}$ photo peak competes favorably with that of 'classical' PMTs, the coincidence time resolution of $1.6 \mathrm{~ns}$ FWHM with dual APD readout is typically lower. This is attributed to the stochastic photon production mechanism in LSO and the photon conversion characteristic of the photo diode, as well as to the fluctuations in photon conversion, albeit the APD's superior quantum efficiency. Also in terms of CT counting speed, the chosen readout principle is limited by the intrinsic light decay in LSO (40 ns) for each impinging X-ray.
\end{abstract}

Index Terms-Avalanche photodiodes (APD), lutetium-oxyorthosilicate (LSO), time-based readout, time-over-threshold discrimination.

\section{INTRODUCTION}

$\mathbf{T}$ HE goal to physically combine photon-counting-CT and PET scans with the same detector head has been of increasing interest in medical imaging because it offers a number of significant advantages to both the patient and the physician [1]-[3]. Such a method also results in cost savings owing to substantial reductions in single tomograph occupancy and simpler CT-PET image fusion. In addition, photon-counting CT has the potential of lower dose examination.

In such a scenario, however, the common photon detection system must not compromise on the specific performance of the two different scan modalities. In other words, a photon detector optimized for high energy $(511 \mathrm{keV})$ gamma rays in PET

Manuscript received February 15, 2008; revised May 30, 2008. Current version published December 04, 2008. This work was supported by the European Commission in the framework of its FP6 program, proposal 505785 (BioCare).

The authors are with the European Organization for Nuclear Research (CERN), CH-1211 Geneva 23, Switzerland (e-mail: francois.powolny@ cern. ch).

Color versions of one or more of the figures in this paper are available online at http://ieeexplore.ieee.org.

Digital Object Identifier 10.1109/TNS.2008.2004036 should work with low energy $(60-100 \mathrm{keV}) \mathrm{X}$-rays from CT. In addition to larger dynamic range coverage, such a combined detector must cope with drastically different event rates from one modality to another, often exceeding order $100 \mathrm{MHz} / \mathrm{mm}^{2}$ in today's CT installations.

Another challenge for detectors to be operated in a multiple mode environment is to yield competitive performance in terms of spatial-, energy- and timing resolution compared with single mode machines.

In the context of BioCare [4], one of the objectives for a combined CT-PET detector was to improve the spatial resolution by applying a fine-grained pixilated front-end layout. This led to the choice of APD arrays where each channel is coupled to an individual LSO pixel. For our tests we have used Hamamatsu S8550 32-channel APD arrays.

An inevitable consequence of such a highly segmented front end is the high density of electronic readout channels congruent with the detector pixel structure. Therefore, to cope with the high detector occupancy and the associated heat dissipation in the confined space of the tomograph, only low-power ASICS with adequate channel density and radiation tolerance can be used.

In order to optimize functionality in line with integration constraints, a key feature of the proposed readout scheme is to derive both energy and time information from a single time digitizer. Instead of using a Constant Fraction Discriminator (CFD) that provides time stamping for coincidence generation, and a signal sampling architecture that extracts time and amplitude information based on the analog signal sampled by a fast ADC, our time-based readout relies entirely on a digital architecture. In this architecture the time stamp is retrieved from the leading edge of a time over threshold or pulse width modulation discriminator and the energy information, proportional to the pulse width, from its falling edge. A single TDC channel then encodes both the time stamp and the energy signal of each event. Thus, such a time-based readout is very compact and robust, for it needs only one discriminator to deliver both time/coincidenceand energy encoding.

Our tests were restricted to a maximum of two channels, read out by a scope, with the aim to characterize the intrinsic performance of the detection and readout system. No specific issues of system integration have been addressed at this stageof our $\mathrm{R}$ \& D yet. On the other hand, it is our aim to combine the front end electronics into one integrated system which is to optimize the readout of PET and CT (see Ch. III A 3). A second step in the design change is the integration of the preamplifier part and the discriminator in a 32-channel ASIC, matching the number of pixels of the APD array. 


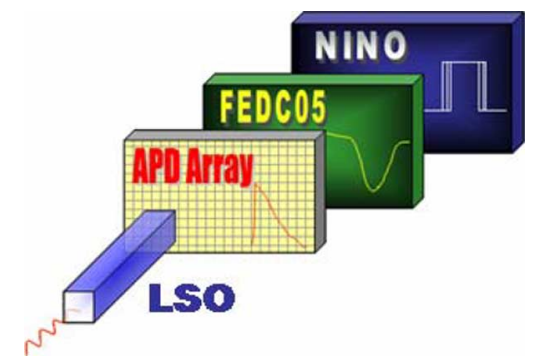

Fig. 1. Schematic of the detector module.

\section{EXPERIMENTAL SETUP}

The schematic of the novel detector architecture is shown in Fig. 1. It consists of a LSO crystal, a 32-channel APD array, a low-noise preamplifier (FEDC05) and an ultra-fast amplifierdiscriminator (NINO).

\section{A. Photo Sensor and Scintillator}

The time based readout system presented in this paper makes use of an avalanche photo diode (APD) coupled to a LSO scintillating crystal [5], [6] typically with dimensions of $2 \times 2 \times 10$ $\mathrm{mm}^{3}$. The chosen photo sensor is the Hamamatsu S8550 APD array [7]-[9] which comprises 32 pixels of $1.6 \times 1.6 \mathrm{~mm}^{2}$ sensitive area each and operating in our tests at a gain of $\mathrm{M}=$ 174. Lower APD gains, together with the chosen discriminator threshold settings, resulted in signal loss for photons of $122 \mathrm{keV}$. The APD's quantum efficiency (QE) is $70 \%$ for $420 \mathrm{~nm}$ photons. The LSO crystals are polished on all faces, wrapped with Teflon tape and mounted 'vertically' (gamma rays incident on the small crystal face) onto the APD with optical grease. Contrary to a horizontal mount, they have a light yield of only 16 photons/keV as measured indepen-dently with a PMT. Scintillators light emission is centered at $420 \mathrm{~nm}$ with a decay time constant of $40 \mathrm{~ns}$.

\section{B. FEDC05}

The FEDC05 front-end circuit [10], implemented in $0.25 \mu \mathrm{m}$ CMOS technology, is a low-noise pulse preamplifier designed for the readout of silicon strip detectors used in the ATLAS experiment [11] at the LHC at CERN. The chip contains 16 channels of charge sensitive amplifiers and analog output drivers. Each channel comprises a fast trans-impedance preamplifier, equipped with an active feedback loop and an integrator circuit, providing $22 \mathrm{~ns}$ peaking time. The output buffer is capable of driving up to $10 \mathrm{pF}$ output load capacitance. The details of the preamplifier and shaper architecture have been published and described in [10].

The detailed noise analysis taking into account all noise sources originating from the input transistor and the feedback circuit is also presented in [10]. For the input transistor biased at $600 \mu \mathrm{A}$, the achievable noise levels (ENC) for detector capacitances of $\sim 10 \mathrm{pF}$ are below $800 \mathrm{e}^{-}$rms.

We intend to operate the front-end amplifier at the lowest possible input charge, i.e., $\mathrm{Q} \geq 1 \mathrm{fC}$ corresponding to $\geq 6250$ electrons in order not to compromise detector efficiency in CT scanning mode and reduce noise hits to negligible levels.

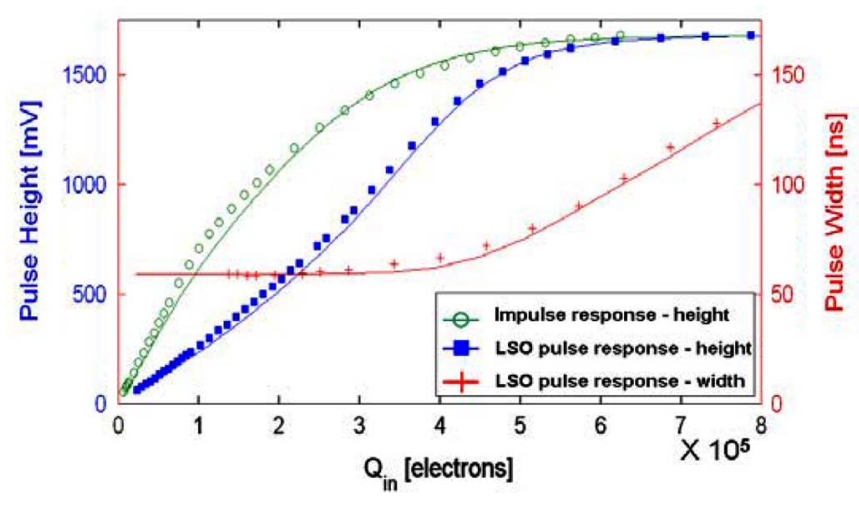

Fig. 2. Calibration of the FEDC05 amplifier: the upper curve (circles) shows the amplifier's impulse response, i.e., to a step-like test pulse, whereas the middle curve (squares) represents the behavior of the amplifier to LSO-like test signals. The bottom curve (crosses) reflects the behavior of the analog pulse width that continues to increase even when the pulse height is fully saturated. Although the agreement between data and SPICE is not $100 \%$, the principal difference in amplifier response to different signal shapes is being reproduced.

The calibration of the chip has been done for two different charge pulse shapes injected at the input of the amplifier. The first one is a test pulse made by sending a voltage step into a $100 \mathrm{fF}$ test capacitance integrated in the preamplifier input, providing the intrinsic impulse response of the electronic channel. The other pulse is generated in such a way that it closely reproduces the timing waveform of the light decay in LSO. It is generated from a voltage step fed into a CR filter with $\tau=C \times R=$ $40 \mathrm{~ns}$. This signal is defined as an LSO-like signal and injected in the input of the FEDC05 chip. Fig. 2 shows the measured response curves of the FEDC05 amplifier to both the test and LSO-like signals (points) and compares them with SPICE simulations (lines). Despite a small deviation of the simulations from the data, SPICE confirms the measured difference in the amplifier's impulse response and that to LSO-like input charges.

The non-linearity of the calibration curves account for the saturation effects in the FEDC05 output stage, however, associated with a gradual increase of the pulse width that continues to grow when the output voltage is saturated, as shown in the 3rd graph of Fig. 2. One can see that for input charges below $1 \times 10^{5}$ electrons the amplifier response to a test pulse is approximately linear in pulse height. For higher charges, i.e., between $1 \times 10^{5}$ and $5 \times 10^{5}$ electrons, the pulse height progressively saturates, while pulse width isincreasing with input charge. For input charges above $5 \times 10^{5}$ electrons the amplifier output is fully saturated with the pulse width increasing almost linearly. In other words, the output signal of the amplifier delivers an output charge that is linear with the input charge.

On the other hand, for LSO-like input pulses, the amplifier response in terms of pulse height stays quasi-linear over a longer range, i.e., for charges up to $4 \times 10^{5}$ electrons. Beyond that, similar saturation effects and increases of pulse width occur as for the test pulses.

It will be shown in the following section that the proportionality of pulse width with input charge directly lends itself to the time-over-threshold technique allowing extracting energy information from a discriminator. 


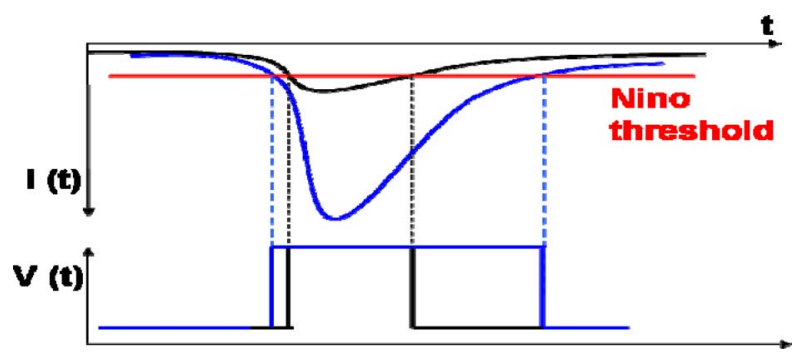

Fig. 3. Schematic of the NINO response to current signals, illustrating the time over threshold technique.

\section{NINO}

The NINO chip [12] is an ultra-fast low-power discrimi-nator developed for the time of flight (TOF) detector of ALICE [13] at the LHC at CERN. It consists of 8 channels and each channel is designed as a fully differential amplifier, a discriminator and an output stage. The amplifier has less than $1 \mathrm{~ns}$ peaking time, and the output pulse a maximum time jitter of $25 \mathrm{ps}$ on the front edge. Each channel consumes $27 \mathrm{~mW}$ of power, and the eight channels fit in a $2 \times 4 \mathrm{~mm}^{2}$ chip processed in IBM $0.25 \mu \mathrm{m}$ CMOS technology.

The NINO discriminator employs the time over threshold technique, i.e., it produces a square pulse with a width proportional to the length of the input pulse above the discriminator threshold (see Fig. 3). This technique, together with the fast timing characteristics of the chip, allows to derive from the pulse width both energy information and very accurate timing. Therefore its high potential for TOF applications in PET.

The discriminator threshold in NINO, variable between 10 and $100 \mu \mathrm{A}$, is too high to sense the APD output current directly. Thus the APD signals need to be amplified with the FEDC05 amplifier to levels compatible with NINO's threshold settings. Interfacing the amplifier with NINO required an external R-C interface $\left(\mathrm{R}_{\text {series }}=4 \mathrm{k} \Omega\right.$ and $\left.\mathrm{C}_{\text {series }}=1 \mathrm{nF}\right)$ for voltage to current transformation, together with chip decoupling and the removal of the DC offset.

The time over threshold technique, however, implies that pulse discrimination of input signals necessarily leads to variations in time walk. Here time walk is understood as the time lag (or delay) between the initial gamma interaction with the crystal and the leading edge of the NINO output signal. Time walk can easily be simulated and tabulated as a function of input charge.

\section{Calibration of the Electronics: FEDCO5 and NINO}

The electronic system is calibrated with LSO-like input signals in order to match realistic conditions as with a LSO scintillator. The electronic system comprises both the FEDC05 and NINO chips mounted on an external PCB and connected via the RC-interface described above.

The response of the combined FEDC05-NINO system to equivalent input charges ranging from $0.5 \times 10^{5}$ to $8 \times 10^{5}$ electrons, is a non-linear relationship between pulse width (NINO output) and input charge. This is shown in Fig. 4, including the SPICE simulation of the system response. This non-linearity is a feature of the NINO stage.

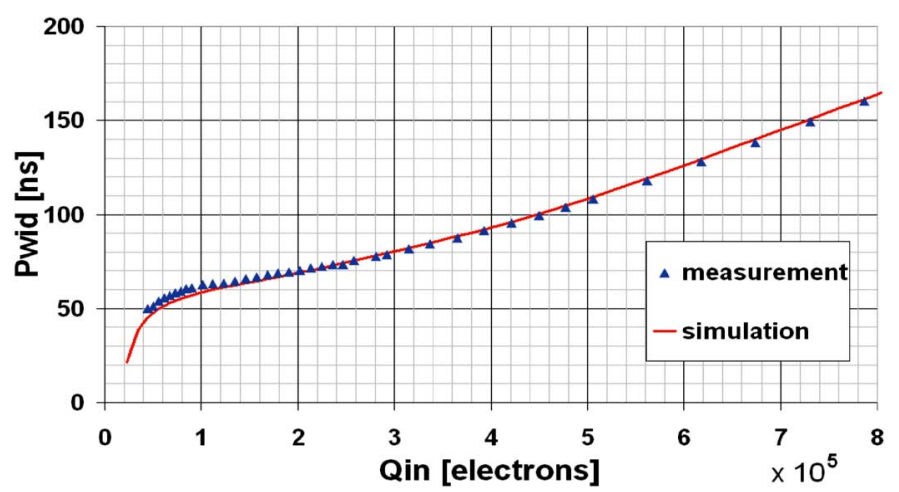

Fig. 4. Pulse width of NINO vs. charge at the input of FEDC05 obtained from calibration measurements (blue triangles) and simulation (red line). Data and simulation show the response of the FEDC05-NINO ensemble to LSO-like pulses. Note: The pulse width results shown here are not the same as those shown in Fig. 2 where the pulse width was measured at half - maximum of the signal coming from the FEDC05 output.

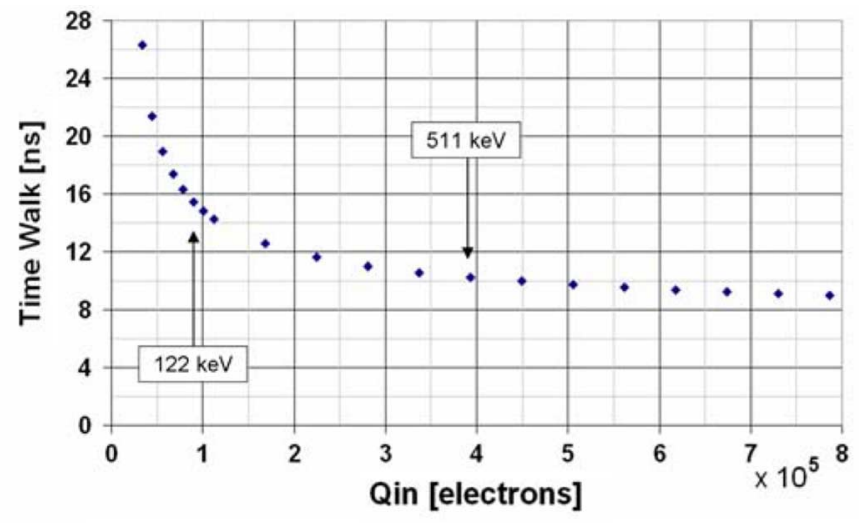

Fig. 5. Simulation of time walk at the output of NINO vs. input charge.

To estimate time walk, similar simulations have been run with the FEDC05-NINO system using LSO-like pulses as input charge. The results, as a function of pulse width, are shown in Fig. 5 .

It is seen from Fig. 5 that the time walk variation between input charges of $1 \times 10^{5}$ electrons, equivalent to the energy of $122 \mathrm{keV}$ CT X-rays, and $3.8 \times 10^{5}$ electrons, corresponding to PET gamma rays, is approximately $5 \mathrm{~ns}$, increasing rapidly towards lower X-ray energies. Furthermore time walk levels off at the pedestal value of $8.5 \mathrm{~ns}$. Thus, at the position of the 511 $\mathrm{keV}$ photo peak, time walk variation per $\mathrm{keV}$ amounts to (10 $\mathrm{ns}-8.5 \mathrm{~ns}) / 511 \mathrm{keV}=3 \mathrm{ps} / \mathrm{keV}$, or $240 \mathrm{ps}$ for a FWHM of 80 $\mathrm{keV}(16 \%)$ of the photo peak (see Ch. III A 1).

\section{ENERGY RESOLUTION}

\section{A. Measurements With Sources}

The detector head that was used for all energy measurements is made of a single LSO crystal with $2 \times 2 \mathrm{~mm}^{2}$ cross section and $10 \mathrm{~mm}$ length, a photo sensor (PMT or APD) and readout electronics specific to the photo sensor. Whereas the PMT is connected to a Multi Channel Analyzer (MCA) for all reference measurements, the APD is read out with the FEDC05-NINO ensemble, and its performance compared with that of the PMT. 


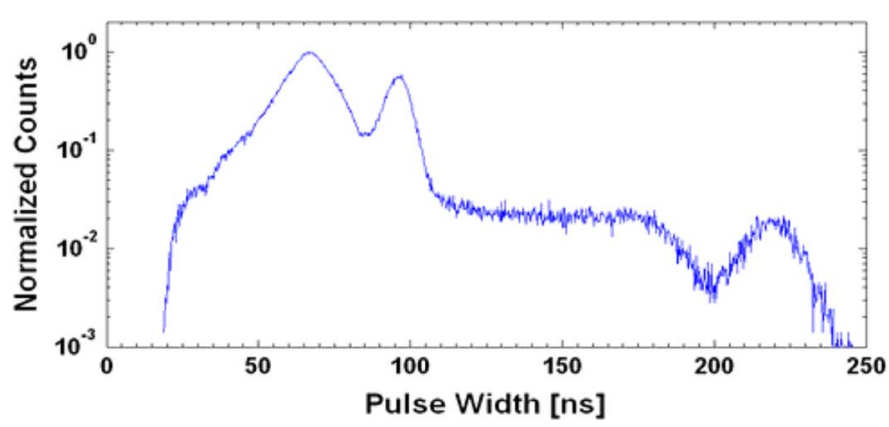

Fig. 6. Frequency plot of the NINO pulse width with the APD detector exposed to a ${ }^{22} \mathrm{Na}$ source.

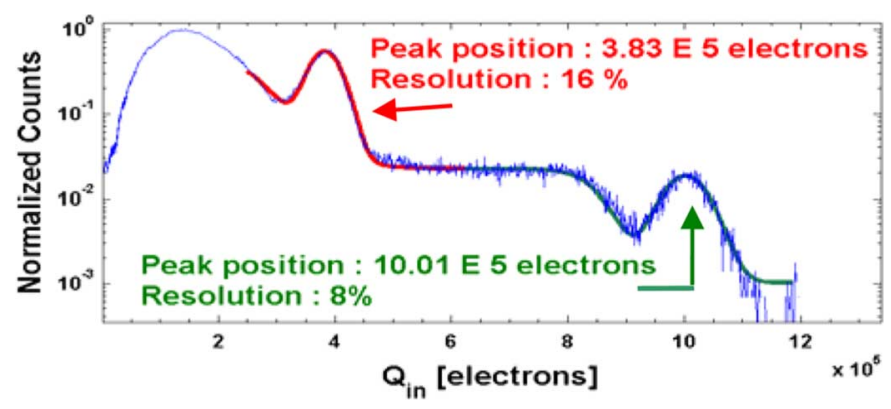

Fig. 7. Rescaled, and normalized to equivalent input charge, histogram taken with the ${ }^{22} \mathrm{Na}$ source and the APD detector head.

Several radioactive sources, such as ${ }^{57} \mathrm{Co},{ }^{22} \mathrm{Na}$ and ${ }^{137} \mathrm{Cs}$, were used allowing us to determine linearity and energy resolution over an energy range of $122 \mathrm{keV} \leq \mathrm{E}_{\gamma} \leq 1275 \mathrm{keV}$.

1) ${ }^{22} \mathrm{Na}$ : Fig. 6 shows a typical ${ }^{22} \mathrm{Na}$ energy spectrum taken with the APD detector head and the FEDC05-NINO electronics. The source has an activity of $2 \mathrm{MBq}$.

This histogram shows the energy spectrum in terms of pulse width [ns] derived from the output of NINO. Since it is known that the FEDC05-NINO ensemble is not linear with input charge (injected into FEDC05), the horizontal scale of the above pulse width histogram is not linear either and has to be normalized to account for that effect. We do this via a look-up table made from prior calibration measurements shown previously in Fig. 3 . The corrected ${ }^{22} \mathrm{Na}$ histogram is shown in Fig. 7.

In this energy spectrum the two photo peaks of ${ }^{22} \mathrm{Na}$ can be seen: one at $511 \mathrm{keV}$ and the other at $1275 \mathrm{keV}$. The photo peak region near $511 \mathrm{keV}$ can be explained as the convolution of three functions: (I) a Gaussian for the photo peak itself, (II) a FermiDirac function that describes the Compton edge associated with the $511 \mathrm{keV}$ peak, and (III) a constant which accounts for the Compton plateau of the second photo peak at $1275 \mathrm{keV}$. Fitting the photo peak region between $2.5 \times 10^{5}$ and $6 \times 10^{5}$ electrons with these three functions permits to derive the mean or peak position of the $511 \mathrm{keV}$ line and determine its energy resolution in FWHM. This was found to be $16 \%$ for the $511 \mathrm{keV}$ photo peak, and, after another fit at $10 \times 10^{5}$ electrons, $8 \%$ for the peak at $1275 \mathrm{keV}$.

2) ${ }^{137}$ Cs: Fig. 8 shows the raw (pulse width) and rescaled (equivalent charge) gamma spectrum from ${ }^{137} \mathrm{Cs}(662 \mathrm{keV}$ at $800 \mathrm{kBq}$ ).

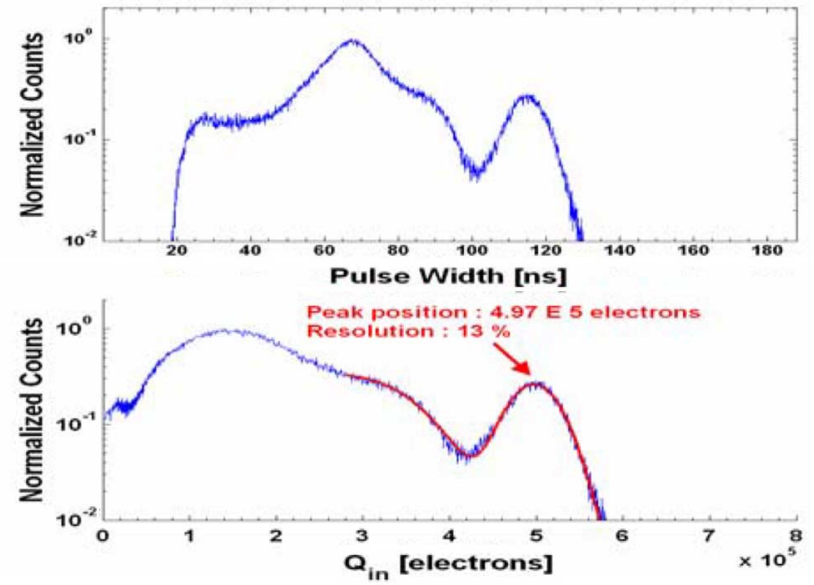

Fig. 8. NINO photon spectra of ${ }^{137} \mathrm{Cs}$ with the APD detector head; top: raw spectrum in terms of pulse width; bottom: spectrum rescaled to equivalent charge.
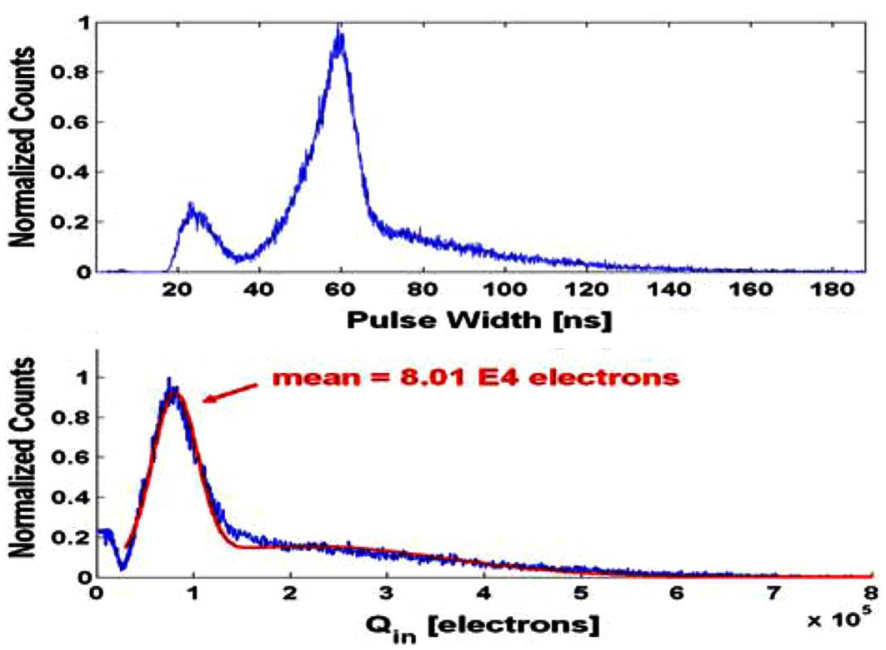

Fig. 9. NINO pulse width spectrum (top) and rescaled spectrum (bottom) of $122 \mathrm{keV} \mathrm{X}$-rays from a ${ }^{57} \mathrm{Co}$ source taken with the APD detector head.

With only one energy line present in this spectrum the fit was done with only two functions, i.e., without the constant function. The energy resolution for the ${ }^{137} \mathrm{Cs}-662 \mathrm{keV}$ line was determined as $13 \%$ FWHM.

3) ${ }^{57} \mathrm{Co}$ : In Fig. 9 we show the unfolded and normalized photon spectra obtained with a ${ }^{57} \mathrm{Co}$ source emitting X-rays at $122 \mathrm{keV}$ and $5 \mathrm{kBq}$.

The composition of this spectrum is different from the previous ones. First, the Compton edge and the backscatter peak are not visible because they are below the NINO threshold. Moreover, the activity of a radioactive lutetium isotope, ${ }^{176} \mathrm{Lu}$, present in the bulk of the LSO crystal becomessignificant because its decay rate is comparable to the ${ }^{57} \mathrm{Co}$ source emission rate.

Due to the onset of the strong nonlinearity in the system's charge/pulse-width relation at input charges below $10^{5}$ electrons (see Fig. 4), coinciding with the peak position of the ${ }^{57} \mathrm{Co}$ line, we are unable to quote a resolution for this energy. All that can be derived from this spectrum is the fact that the $122 \mathrm{keV}$ line is well separated from the noise. 


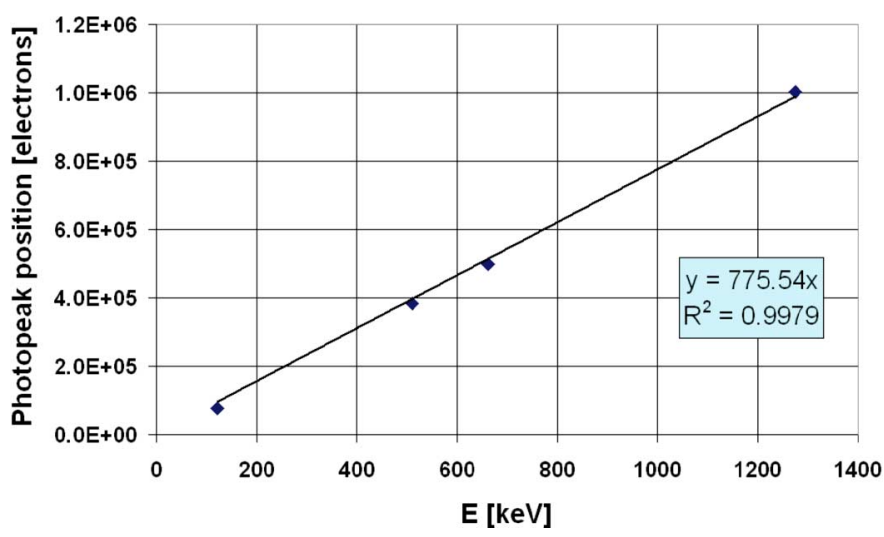

Fig. 10. Correlation between photon energy and equivalent charge by plotting the photo peak position [electrons] versus the source energy.

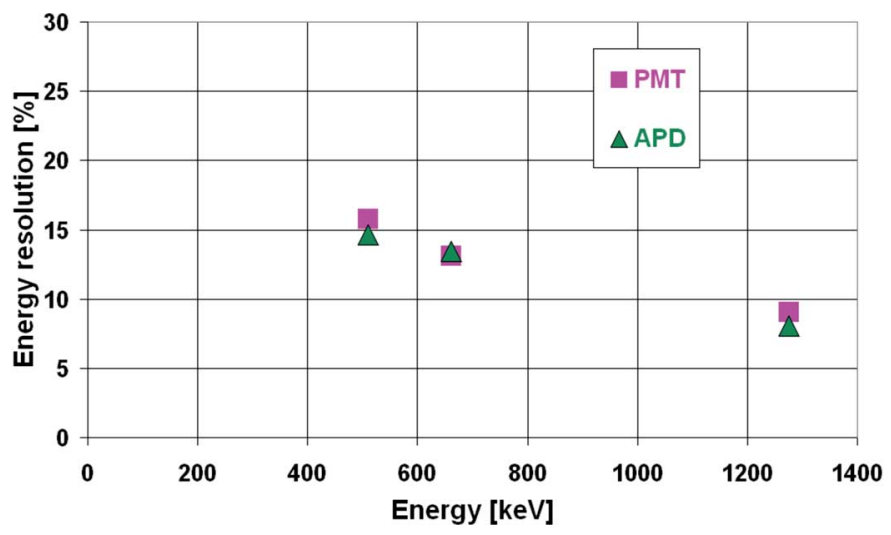

Fig. 11. Comparison of energy resolution obtained with two different detection systems. Results with classical PMT-ADC readout are shown as squares; those from the APD-FEDC05-NINO time based readout are given as triangles.

This applies also to lower X-ray energies and is a potential problem with this particular setup. Therefore, for an integrated PET/CT readout and specifically aiming to improve on the low energy CT detection, the ASIC design will be adapted to overcome the strong non-linearity of NINO, a feature exploi-ted for its primary application in multi-parallel resistive plate chambers (RPC) [13]. This would then yield a linear pulse width as a function of input charge and make the detection of CT X-rays much easier.

\section{B. Data Analysis and Results}

The photo peak position derived from the source spectra is plotted versus the source energies and shown in Fig. 10.

From the fit, the four data points scale linearly with zero intercept. This is to be expected since the pedestals in the normalized energy spectra are zero. Fig. 10 then demonstrates the viability of our normalization technique that was developed to eliminate the inherently non-linear effects of the system components.

In order to compare the energy resolution obtained with the time-based setup, we took as benchmark a classical PMT read out with a MCA using the same scintillating crystals from the APD setup. It should be noted that, in order to suppress temperature induced gain fluctuations, the APDs were kept at a constant temperature of $20 \pm 0.5^{\circ} \mathrm{C}$. The results are shown in Fig. 11 .
TABLE I

Properties of Three DifFERENT SCINTILlators (SEe Ch. II A For CONDITIONS OF MEASUREMENT)

\begin{tabular}{cccc}
\hline \hline & $\begin{array}{c}\text { Crystal } \\
\text { composition }\end{array}$ & $\begin{array}{c}\text { Dimensions } \\
{\left[\mathrm{mm}^{3}\right]}\end{array}$ & $\begin{array}{c}\text { Light yield } \\
\text { [photons/MeV] }\end{array}$ \\
\hline C1 & LSO & $2 \times 2 \times 10$ & 16,100 \\
C2 & LSO & $2 \times 2 \times 10$ & 14,400 \\
C3 & LSO & $2 \times 2 \times 15$ & 16,600 \\
\hline
\end{tabular}

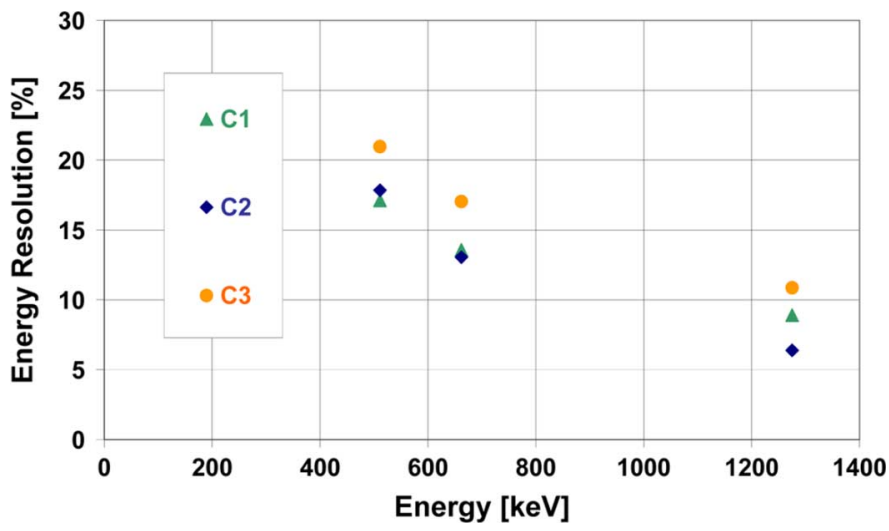

Fig. 12. Performance of three different crystals in terms of energy resolution using the APD time-based readout.

With the exception of the $122 \mathrm{keV}$ X-ray line whose width could not be determined, the resolution of the other source energies has comparable values for both the PMT-ADC and the APD-FEDC05-NINO system. In fact, one can expect the APD's advantage of a higher quantum efficiency and hence superior energy resolution being partially offset by the APD/LSO mismatch in sensitive area of $(1.6 \mathrm{~mm})^{2} /(2 \mathrm{~mm})^{2}$ and the addition of front end electronics noise, both not present in the PMT setup.

The same method has also been applied to different crystals. Their characteristic features are shown in Table I.

All tested crystals have the same cross-section of $2 \times 2 \mathrm{~mm}^{2}$, close to that of the APD pixel, however, with two different lengths, i.e., $10 \mathrm{~mm}$ and $15 \mathrm{~mm}$. $\mathrm{C} 1$ and $\mathrm{C} 2$ have identical dimensions but are from different manufacturers. Fig. 12 shows the energy resolution obtained with each of them.

\section{Coincidence Time Resolution}

After having shown the viability of the time-based readout to provide good energy resolution comparable with PMT-based systems, the technique must also be evaluated on the grounds of timing resolution itself. This feature is especially important in PET where image quality greatly benefits from the ability to detect two collinear photons within a tight time window. This necessitates high-precision coincidence mea-surements between two opposite detector heads, eventually allowing event selection via TOF.

In the following sections we will evaluate the timing per-formance of the chosen APD-based system. The figure of merit for this type of performance is defined as coincidence time resolution, also called time jitter.

\section{A. Reference Measurement: PMT vs. PMT}

The most commonly used standard for photon detection with highest timing precision is still the PMT with its 


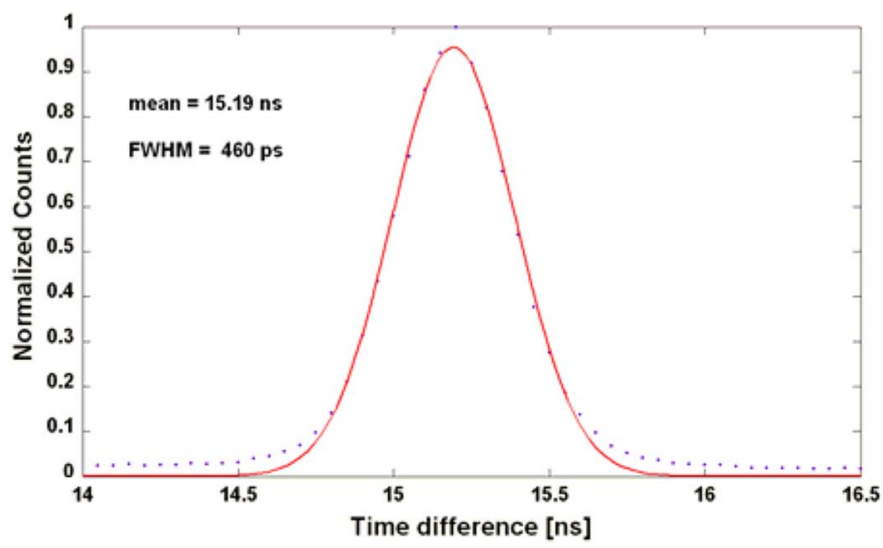

Fig. 13. Time difference histogram of the two reference PMTs in coincidence.

sub-nanosecond rise time and transit times. We have thus chosen as reference photo-detector the Hamamatsu 6533 PMT with a rise time of $0.7 \mathrm{~ns}$ and a transit time spread of $0.16 \mathrm{~ns}$ FWHM [14]. This PMT has a diameter of $25 \mathrm{~mm}$ and contains ten dynode stages and a bi-alkali photocathode.

Two such PMTs are mounted back-to-back, and a ${ }^{22} \mathrm{Na}$ source is installed in the middle of them. Each PMT has one LSO crystal of $2 \times 2 \times 10 \mathrm{~mm}^{3}$ mounted centrally on its entrance window coupled with silicon grease. A constant fraction discriminator (CFD) is connected to each PMT output. Thus, the coincidence measurements with this setup are free of time walk. The CFD outputs, both 50 ns long, are read out and processed by a fast digital oscilloscope, a technique commonly used for this type of measurements [15].

In the following we define coincidence time resolution as the variation of the time difference measured between the two front edges of the CFD outputs. One of the CFD outputs is also chosen to form a gate. A fixed delay of $15 \mathrm{~ns}$ is added to the other CFD output. Events are gated in such a way that the arrival of collinear photons must fall into a $50 \mathrm{~ns}$ window. Other events are rejected.

A histogram of the two PMT-CFDs' arrival time difference is shown in Fig. 13.

The distribution is fitted with a Gaussian having a FWHMof close to 500 ps.

\section{B. Coincidence Measurement: PMT vs. APD}

In the new setup we replaced one of the PMT-CFD detector heads with the APD-FEDC05-NINO detector system. In this arrangement, the time difference from the coincidence measurements is made between the CFD front edge and the NINO leading edge, the latter not being free of time walk. Therefore, for each event, the pulse width information of NINO needs to be corrected for time walk.

Fig. 14(a) shows what happens if one plots the time difference against the corresponding pulse width. This leads to a typical scatter plot in which timing and energy information are strictly correlated. In other words, if the data are projected on the horizontal axis of the scatter graph one derives the ${ }^{22} \mathrm{Na}$ energy spectrum, though without the $1275 \mathrm{keV}$ line produced by non-correlated photons [Fig. 14(b)]. On the other hand, projecting the
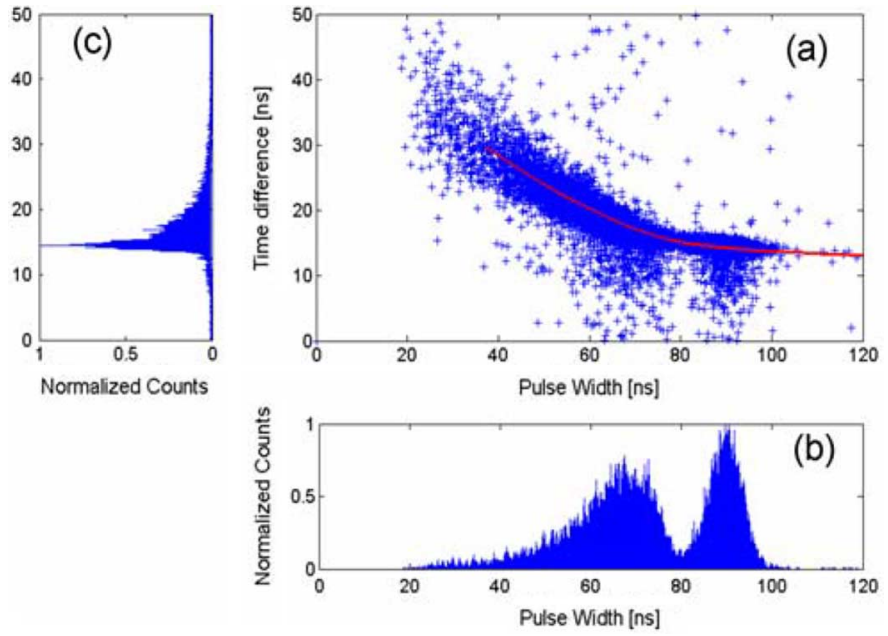

Fig. 14. Correlation of pulse width and time difference for coincident photons from ${ }^{22} \mathrm{Na}$ in a PMT vs. APD setup (a). The projections yield the corresponding energy spectrum (b, horizontal) and the time difference spectrum (c, vertical).

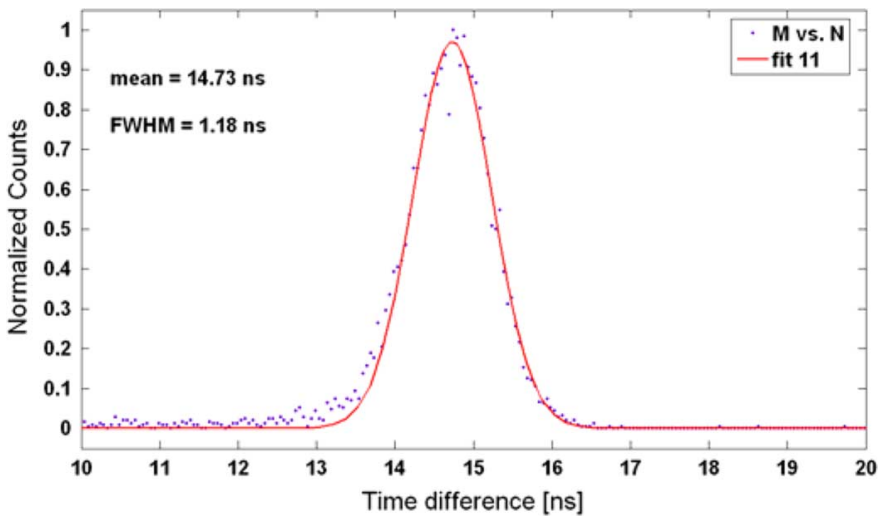

Fig. 15. Time difference histogram from the PMT vs. APD measurements. The spectrum is corrected for time walk in NINO and constrained to events in the ${ }^{22} \mathrm{Na}$ photo peak region.

data entries on the vertical axis reproduces the associated time difference histogram from coincident gamma rays [Fig. 14(c)].

Embedded in the time-energy scatter graph is also the trend of time walk to increase towards lower energies, here expressed in terms of pulse width. The previously simulated time walk relation as a function of input charge or energy (see Fig. 5) is superimposed on the data and shown as a continuous line. The vertical offset is due to cable delays.

What is also seen from the time difference histogram (vertical projection) is the effect of time walk spreading the distribution towards larger time intervals. Thus, the necessity to remove the effect of time walk by applying, event-by-event, the proper corrections. These corrections, in the form of a look-up table, were derived from the simulations described in Chapter II, D and shown previously in Fig. 5.

To further improve on timing precision we have only selected events around the $511 \mathrm{keV}$ line, thus eliminating Compton and backscatter events. The time difference spectrum, now time walk corrected and constrained to the photo peak, is shown in Fig. 15. 
From the fit of the nearly Gaussian distribution in Fig. 15 we determine a standard deviation $\sigma_{\mathrm{TD}}$ of $500 \mathrm{ps}$. $\sigma_{\mathrm{TD}}$ is convoluted by the individual time resolution of the PMT setup and the APD-NINO system, hence given as:

$$
\sigma_{\mathrm{TD}}=\sqrt{\sigma_{\mathrm{APD}}^{2}+\sigma_{\mathrm{PMT}}^{2}} .
$$

From the previously made reference measurements in which two identical PMTs were used (see Chapter IV, A) we can extract the intrinsic single-PMT time resolution $\sigma_{\mathrm{PMT}}$ from:

$$
\sigma_{\mathrm{PMT}}=\frac{200}{\sqrt{2}}=141 \mathrm{ps} .
$$

Replacing $\sigma_{\mathrm{PMT}}$ in (1) with the expression (2) delivers the intrinsic APD-NINO time resolution $\sigma_{\mathrm{APD}}$ :

$$
\begin{aligned}
& \sigma_{\mathrm{APD}}=480 \mathrm{ps} \\
& \text { or } \\
& \mathrm{R}_{\mathrm{APD}}=1.13 \mathrm{~ns} \text { FWHM. }
\end{aligned}
$$

\section{Coincidence Measurement: APD vs. APD}

The ultimate objective is to show how the timing performance of an all-APD-based system such as ours compares with that of the commonly used high speed PMT detectors in PET. Therefore the coincidence setup was modified in order to accommodate two APD detectors mounted back-to-back, running both with our FEDC05-NINO-electronics. No CFD but only leading edge discrimination is used, implying that both coincidence signals are smeared by time walk variations. Thus, we must record pulse width and time stamp for both APD detectors. Otherwise, the principle of measurement is the same as before, except that one of the NINO output signals is used to generate a gate of 150 ns.

To account for the intrinsic time jitter of the gate itself, two time difference measurements must be made, i.e., recording the time between the front edge of the gate and each leading edge of the two NINO outputs. Subtraction of the two time intervals then yields the true mutual coincidence time difference between the two APD-NINO outputs, independent of the gate.

In Fig. 16 we show how the raw coincidence time spectrum of an APD system improves by applying successively photopeak selection and time walk corrections on each signal output (from top to bottom).

The bottom spectrum of Fig. 16 is fitted with a Gaussian and shown separately in Fig. 17.

From the fit, the standard deviation $\sigma_{\text {system }}$ of the dual APD setup is found to be $650 \mathrm{ps}$, or $\leq 1.6 \mathrm{~ns}$ FWHM.

This result proves our earlier measurements with a single APD detector correct, since

$$
\begin{aligned}
\sigma_{\text {System }} & =\sqrt{\sigma_{\mathrm{APD} 1}^{2}+\sigma_{\mathrm{APD} 2}^{2}} \\
& \sim \sqrt{2 \times(480 \mathrm{ps})^{2}}=680 \mathrm{ps}, \\
\text { or } \leq & 1.6 \mathrm{~ns} \mathrm{FWHM.}
\end{aligned}
$$

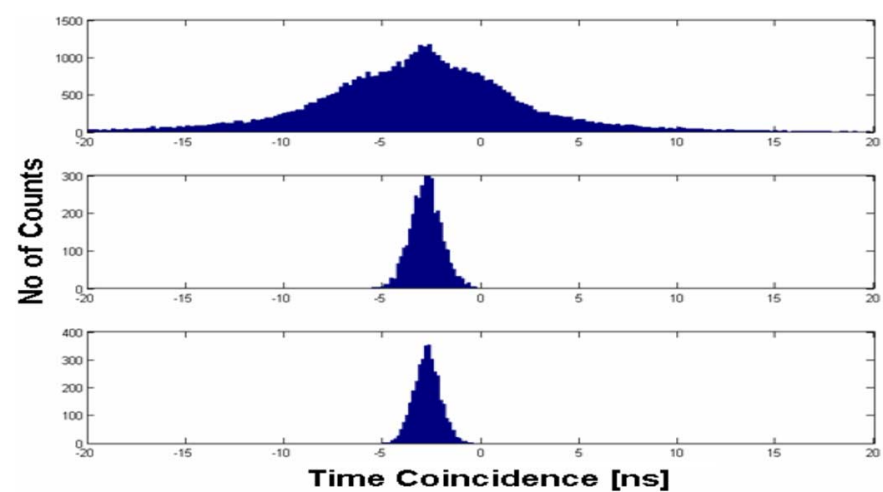

Fig. 16. Coincidence time spectra of a double APD-NINO system: Top: raw spectrum; Middle: Spectrum after applying a photo peak constraint; Bottom: Spectrum further refined by time walk corrections on the remaining data sample.

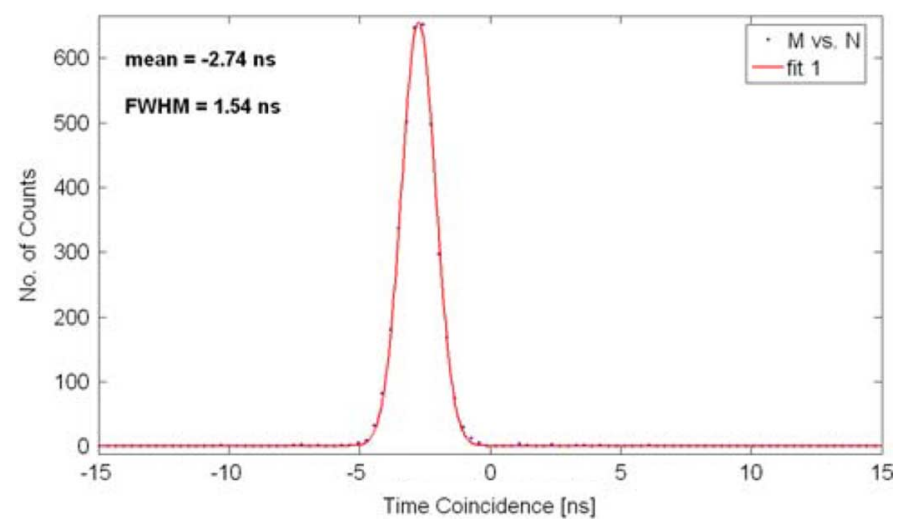

Fig. 17. Fit of the refined time coincidence spectrum of two APD detectors.

Under the assumption that, despite data correction and refinement, some 'fake' coincidence events are still left in the data sample, we repeated some coincidence measurements at a larger distance from the source. Table II is a summary of our results and shows three geometric scenarios for three different detector combinations $\left(\mathrm{PMT}_{1} / \mathrm{PMT}_{2}, \mathrm{PMT}_{1} / \mathrm{APD}_{1}\right.$ and $\left.\mathrm{PMT}_{1} / \mathrm{APD}_{2}\right)$. Zero-distancedenotes closest spacing between detector and source, whereas ' $5-10 \mathrm{~cm}$ ' describes an asym-metric arrangement where one detector is $5 \mathrm{~cm}$ and the other $10 \mathrm{~cm}$ away from the source.

The results in Table II show that different detector-source distances have little influence on the achievable time resolution. We can hence conclude that the intrinsic time resolution $\mathrm{R}_{\mathrm{APD}}$ of the APD-FEDC05-NINO ensemble is:

$$
\mathrm{R}_{\mathrm{APD}} \leq 1.2 \mathrm{~ns} \text { FWHM, }
$$

or for the combined dual APD system:

$$
\mathrm{R}_{\text {System }} \leq 1.6 \text { ns FWHM. }
$$

From [16]-[18], it turns out that our time-based approach of reading and processing coincident photon data from an APD circuit is comparable to classical methods where APD signals are digitized with ADCs. However, timing resolution of the order of $1 \mathrm{~ns}$ is relatively poor when electronic circuits with significantly 
TABLE II

Summary of COINCIDENCE MEASUREMENTS With DiFFERENT DETECTOR CONFIGURATIONS

\begin{tabular}{|c|c|c|c|c|c|}
\hline \multicolumn{3}{|c|}{ Distance source/detector: $[\mathrm{cm}]$} & $\mathbf{0}$ & 5 & $5-10$ \\
\hline \multirow{2}{*}{$\begin{array}{c}\text { PMT } \\
\text { vs. } \\
\text { PMT }\end{array}$} & & $\sigma[\mathrm{ns}]$ & 0.20 & 0.19 & 0.19 \\
\hline & & $\mathrm{IM}[\mathrm{ns}]$ & 0.46 & 0.44 & 0.44 \\
\hline \multirow{4}{*}{$\begin{array}{l}\text { PMT } \\
\text { vs. } \\
\text { APD }\end{array}$} & Intrinsic $A$ & $1: \sigma[\mathrm{ns}]$ & 0.43 & 0.45 & 0.45 \\
\hline & & $\mathrm{IM}[\mathrm{ns}]$ & 1.01 & 1.06 & 1.05 \\
\hline & Intrinsic APD2: & $\sigma[\mathrm{ns}]$ & 0.48 & 0.48 & 0.49 \\
\hline & & $\mathrm{IM}[\mathrm{ns}]$ & 1.13 & 1.13 & 1.14 \\
\hline
\end{tabular}

better timing characteristics, i.e., lower time jitter, are used. It is thus worthwhile to investigate the different sources of time jitter that contribute to the overall system time resolution.

In an approach similar to the one by Casey et al. [19] we factorize the overall timing uncertainty into three parts corresponding to the three detector components in our system, each contributing to time jitter: (I) the scintillating crystal, (II) the APD photodetector, and (III) the electronic circuit. Statistically, the time jitter can then be expressed as the quadratic sum of the three contributions:

$$
\sigma_{j \text { Total }}=\sqrt{\sigma_{j \text { Crystal }}^{2}+\sigma_{j \text { APD }}^{2}+\sigma_{j \text { Electr }}^{2}}
$$

where: $\sigma_{j}$ Crystal denotes jitter coming from the scintillating crystal, $\sigma_{j}$ APD that of the APD and $\sigma_{j}$ Electr the electronic contribution to time jitter.

1) Time Jitter Produced by Electronic Noise: Considering the electronic part of the detector alone, time precision is only governed by electronic noise. In fact, the instantaneous signal level produced at the output of FEDC05 is modulated by noise $\sigma_{\mathrm{e}}$. This makes the time of threshold crossing fluctuate. The electronic time jitter $\sigma_{j \text { Electr }}$ is then given by the ratio of the amplifier noise $\sigma_{\mathrm{e}}$ and the signal $(d V / d t)$ delivered within the rise time of the amplifier at the discriminator threshold crossing (also denoted as slope to noise ratio) [20]:

$$
\sigma_{j \text { Electr }}=\sigma_{e} \times\left(\frac{d V}{d t}\right)_{\text {Threshold }}^{-1} .
$$

This time jitter is evaluated by injecting test charges at the input of the amplifier (FEDC05) and by measuring the corresponding signal delays at the output of NINO. Since input or detector capacitances influence front-end noise, our measurements were made with the fully biased APD array coupled to the FEDC05 amplifier. This leads to approximately $10 \mathrm{pF}$ at the input of FEDC05 [10]. In addition, the APD dark current also adds to the noise at the input of the amplifier. Thus, $\sigma_{j}$ Electr is a convolution of the intrinsic amplifier front-end noise and the two noise contributions from the biased APD. The electronic time jitter $\sigma_{j}$ Electr, measured under these conditions, is plotted in Fig. 18. One should note that the steep decline in time jitter for low input charges and its asymptotic behavior for charges

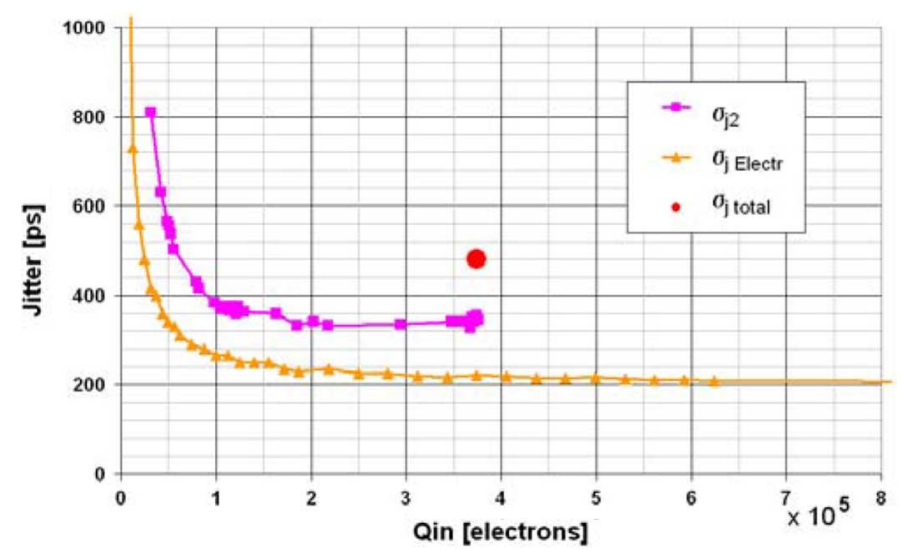

Fig. 18. Different measured time jitters $\sigma_{j \text { Electr }}, \sigma_{j 2}$, and $\sigma_{j \text { total }}$ versus input charge $\mathrm{Q}_{\text {in }}$ in FEDC05. Note, $\sigma_{j \text { total }}$ is only one point as it denotes the total jitter of the system measured with only one gamma energy of $511 \mathrm{keV}$ from ${ }^{22} \mathrm{Na}$.

$>2 \times 10^{5}$ electrons is only a consequence of the previously described output signal behavior of FEDC05. It was shown then that the output signal of FEDC05 rapidly rises with increasing input charge $(d V / d t \gg 1)$ and then gradually reaches full saturation (with increasing pulse length) where the pulse rise time, however, does not increase anymore, i.e., $d V / d t \sim$ const.

2) Time Jitter From the APD Amplification Mechanism: APD signal amplification through avalanche processes underlies device-specific fluctuations also leading to time variations of the output signals. To test this mechanism and its influence on time jitter we have sent laser light bursts of a defined number of photons of $410 \mathrm{~nm}$ onto the APD window. The resulting time jitter $\sigma_{j 2}$, also shown in Fig. 18, is now a convolution of the time variations produced by the avalanche mechanism of the APD and the electronic time jitter $\sigma_{j}$ Electr described above:

$$
\sigma_{j 2}=\sqrt{\sigma_{j \text { Electr }}^{2}+\sigma_{j \mathrm{APD}}^{2}}
$$

3) Overall System Time Jitter: The overall system time jitter, $\sigma_{j \text { total }}$, i.e., the one made of all components described above, plus the jitter induced by the non-uniform light production of the scintillating crystal, was measured with $511 \mathrm{keV}$ gammas from ${ }^{22} \mathrm{Na}$ and is also shown in Fig. 18, as a single point only, in fair agreement with [19].

To compute the individual jitter contributions in (5) we concentrate only on the charge equivalent to the $511 \mathrm{keV}$ photo peak, i.e., $3.8 \times 10^{5}$ electrons, which is common to all three measurements. Hence, this is the only region where the three effects of jitter can be deconvoluted.

From SPICE, and using the nominal input charge of $3.8 \times 10^{5}$ electrons, we have determined the signal rise of the FEDC05 output signal at threshold crossing as:

$$
d V / d t=33 \mathrm{mV} / \mathrm{ns} .
$$

And the equivalent noise voltage was measured as

$$
\sigma_{V}=8 \mathrm{mV} .
$$


TABLE III

JiTter COMPONENTS IN THE TIME BASED SYSTEM

\begin{tabular}{ccccc}
\hline \hline Contribution & Electronic & APD & Crystal & Total \\
\hline$\sigma_{\mathrm{jitter}}[\mathrm{ps}]$ & 220 & 230 & 340 & 480 \\
{$[\%]$} & 20 & 30 & 50 & 100 \\
\hline \hline
\end{tabular}

Thus, from (6) we derive:

$$
\begin{aligned}
\sigma_{j \text { Electr }} & =\sigma_{e} \times\left(\frac{d V}{d t}\right)_{\text {Threshold }}^{-1} \\
& =8 \times(33)^{-1}=242 \mathrm{ps}
\end{aligned}
$$

in good agreement with the measured asymptotic value of $\sigma_{j}$ Electr shown in Fig. 18:

$$
\sigma_{j \text { Electr }}^{\text {asymptic }} \approx 220 \text { ps. }
$$

The equivalent noise charge at the input of the amplifier is $\sim 1400$ electrons rms, in fair agreement with [10].

The jitter attributed to the APD amplification mechanism is extracted from Fig. 18 where $\sigma_{j 2}=340$ ps; using expression (7), APD jitter becomes:

$$
\sigma_{j \mathrm{APD}}=230 \mathrm{ps} .
$$

Finally, we are able to estimate the crystal jitter $\sigma_{j}$ Crystal by making use of (5) and expressions (8) and (9) knowing that the overall system jitter was measured as 480 ps with $511 \mathrm{keV}$ photons from ${ }^{22} \mathrm{Na}$ (Chapter IV, B):

$$
\sigma_{j \text { Crystal }}=340 \mathrm{ps} .
$$

The total time jitter, its components, and their relative contribution to the total are summarized in Table III.

The results demonstrate that the noise of the electronics developed at CERN, combined with the noise induced by the APD, is $20 \%$ of the total jitter. Non-uniformities in avalanche amplification inside the APD account for $30 \%$ of the total jitter. The effect is explained by the excess noise factor (ENF) in the APD, which describes the variation of electron multiplication during the avalanche process.

The final contribution of $50 \%$ to the system time jitter is seen to be due to a combination of two effects: It is not only associated with the Poisson-like light production in the crystal within the $40 \mathrm{~ns}$ decay time, but also by the lacking gain of the APD rendering the system insensitive to fewer than $\sim 20$ photoelectrons [21]. Owing to the high gain of PMTs the stochastic nature of light production in the crystal, and hence its induced jitter, are nearly completely offset by the PMT's ability to amplify single photoelectrons. Our measurements have confirmed this effect showing a PMT-based system-time- jitter of only 200 ps.

\section{CONCLUSION}

We have shown that, in terms of energy resolution, efficiency and signal to noise ratio, a purely time-based readout technique for PET and CT photons, coupled to adequate high speed and low noise electronics, competes very well with classical readout methods. In addition, our system offers numerous advantages over standard architectures, such as higher reliability, simplicity, and easier system integration. This has a direct and positive impact on spatial resolution and cost when highly segmented or pixilated photon detectors are chosen for tomographs.

Timing precision, however, is severely limited by the use of APDs as long as crystals with rather long light decay characteristics and modest light yield are used. In the case of LSO, a rather common and widely used crystal in medical imaging, time resolution in conjunction with the used Hamamatsu-S8550 APDs is inherently limited to $1.6 \mathrm{~ns}$ FWHM, independent of the electronics used.

These results nonetheless suggest other and new choices of photodetectors than PMTs, offering the advantages of high pixel density together with higher gain. Silicon Photo-Multipliers (SiPM) are such possibilities whose signal could be sensed by the NINO discriminator directly, i.e., without an additional amplifier in the chain. Such an approach would further simplify the readout architecture and system integration, and finally overcome the inherently large timing fluctuations imposed by LSO-APD detector heads.

\section{ACKNOWLEDGMENT}

The authors would like to express their deep gratitude to H.-F. Hoffmann and M. Dosanjh for their continued and firm support throughout the duration of the work. The authors are equally grateful to M. Despeisse and A. Nardulli for their help and many enlightening discussions in the course of the work presented here; J. Trummer and B. Frisch for the characterization of the crystals; and F. Anghinolfi and J. Kaplon for their invaluable help in the electronics.

\section{REFERENCES}

[1] D. W. Townsend et al., "PET/CT today and tomorrow," J. Nucl. Med., vol. 45, no. 1, pp. 4-14, 2004

[2] R. Fontaine et al., "Architecture of a dual-modality, high-resolution, fully digital positron emission tomography/computed tomography (PET/CT) scanner for small animal imaging," IEEE Trans. Nucl. Sci., vol. 52, no. 3, pp. 691-696, Jun. 2005.

[3] A. Nassalski et al., "The road to the common PET/CT detector," IEEE Trans. Nucl. Sci., vol. 54, no. 5, pp. 1464-1473, Oct. 2007.

[4] BioCare (Molecular Imaging for Biologically Optimized Cancer Therapy), European Commission 6th Framework, Proposal No. 505785 [Online]. Available: http://www.biocare-eu.com/home. aspx? $\mathrm{r}=4 \& \mathrm{~s}=1 \& \mathrm{ss}=$

[5] H. Suzuki et al., "Light emission mechanism of $\mathrm{Lu}_{2}\left(\mathrm{SiO}_{4}\right) \mathrm{O}: \mathrm{Ce}$," IEEE Trans. Nucl. Sci., vol. 40, no. 4, pp. 380-383, Aug. 1993.

[6] M. J. Weber, "Scintillation: Mechanisms and new crystals," Nucl. Instrum. Methods Phys. Res. A, vol. A527, pp. 9-14, 2004.

[7] Hamamatsu [Online]. Available: http://jp.hamamatsu.com/products/sensor-ssd/pd078/pd079/pd085/S8550/index_en.html

[8] J. Ch. Vanel, "Characterization of avalanche photodiodes for calorimetry applications," Nucl. Instrum. Methods Phys. Res. A, vol. A428, pp. 413-431, 1999.

[9] M. Kapusta et al., "Hamamatsu S8550 APD arrays for high-resolution scintillator matrices readout," Nucl. Instrum. Methods Phys. Res. A, vol. A504, pp. 139-142, 2003.

[10] J. Kaplon et al., "Fast CMOS binary front end for silicon strip detectors at LHC experiments," IEEE Trans. Nucl. Sci., vol. 52, no. 6, pp. 2713-2720, Dec. 2005

[11] ATLAS Collaboration [Online]. Available: http://atlas.web.cern.ch/ Atlas/index.html

[12] F. Anghinolfi et al., "NINO: An ultrafast low-power front-end amplifier discriminator for the time-of-flight detector in the ALICE experiment," IEEE Trans. Nucl. Sci., vol. 51, no. 5, pp. 1974-1978, Oct. 2004. 
[13] ALICE Collaboration [Online]. Available: http://aliceinfo.cern.ch

[14] Hamamatsu [Online]. Available: http://jp.hamamatsu.com/products/sensor-etd/pd002/pd394/H6533/index_en.html

[15] W. W. Moses, "Prospect for time-of-flight PET using LSO scintillators," IEEE Trans. Nucl. Sci., vol. 46, no. 3, pp. 474-478, Jun. 1999.

[16] B. J. Pichler et al., "Lutetium oxyorthosilicate block detector readout by avalanche photodiode arrays for high resolution animal PET,' Phys. Med. Biol., vol. 49, pp. 4305-4319, Sep. 2004.

[17] S. McCallum, "A four-layer attenuation compensated PET detector based on APD arrays without discrete crystal elements," Phys. Med. Biol., vol. 50, pp. 4187-4207, Aug. 2005.
[18] C. Pepin et al., "Properties of LYSO and recent LSO scintillators for phoswich PET detectors," IEEE Trans. Nucl. Sci., vol. 51, no. 3, pp. 789-795, Jun. 2004.

[19] M. E. Casey et al., "Analysis of timing performance for an APD-LSO scintillation detector," Nucl. Instrum. Methods Phys. Res. A, vol. A504, pp. 143-148, 2003.

[20] H. Spieler, Semiconductor Detector Systems. London, U.K.: Oxford Univ. Press, 2005.

[21] F. J. Lynch, "Improved timing with NaI(T1)," IEEE Trans. Nucl. Sci., vol. 13, no. 3, pp. 140-147, Jun. 1966. 